\title{
Investigation of Pseudomonas aeruginosa Resistance Pattern against Antibiotics in Clinical Samples from Iranian Educational Hospital
}

\author{
Zakieh Rostamzadeh $^{1}$, Mahshid Mohammadian², Alireza Rostamzade ${ }^{3}$ \\ ${ }^{1}$ Solid Tumor Research Center, Urmia University of Medical Science, Urmia, Iran \\ ${ }^{2}$ Department of Biochemistry, School of Medicine, Urmia University of Medical Sciences, Urmia, Iran \\ ${ }^{3}$ Cardiology Department, Seidoshohsda Hospital, Urmia University of Medical Sciences, Urmia, Iran \\ Email: "tarjomeh4u@gmail.com
}

Received 4 August 2016; accepted 14 March 2016; published 17 March 2016

Copyright (C) 2016 by authors and Scientific Research Publishing Inc.

This work is licensed under the Creative Commons Attribution International License (CC BY).

http://creativecommons.org/licenses/by/4.0/

(c) (i) Open Access

\begin{abstract}
Pseudomonas aeruginosa (P. aeroginosa) is one of the opportunistic pathogens, which is the main cause of prevalent hospital infections worldwide. The aim of this study was to determine the prevalence of antibiotic resistance pattern against $P$. aeroginosa from clinical samples in our population. This study was performed during March 2009 to September 2011. During this period 233 clinical isolated samples from hospital patients were examined. In these studies, different strains of $P$. aeroginosa were isolated from samples, then microbiologically tested. Bacterial susceptibility was performed by the disc-diffusion tests with Kirby Baur disc diffusion tests in Muller-Hinto environment. Our results showed maximum antibiotic resistance $(99.5 \%)$ of $P$. aeruginosa against Trimetoprime Solfametoxasole and Ciprofloxacin (55.33\%), Amikacin (61\%), Imipenem (33\%), which were identified as the most effective antibiotics in this study. In conclusion, indeed most Pseudomonas aeruginosa strains infections are treated as soon as possible due to their severe resistance against antibiotics. So, we have to apply an accurate antibiotic treatment discipline, according to the finding, based on antibiogram, in order to prevent its spread and also, monitoring and optimization of antimicrobial use should be considered carefully.
\end{abstract}

\section{Keywords}

Pseudomonas aeruginosa, Resistance, Antibiotics

\footnotetext{
${ }^{*}$ Corresponding author.
}

How to cite this paper: Rostamzadeh, Z., Mohammadian, M. and Rostamzade, A. (2016) Investigation of Pseudomonas aeruginosa Resistance Pattern against Antibiotics in Clinical Samples from Iranian Educational Hospital. Advances in Microbiology, 6, 190-194. http://dx.doi.org/10.4236/aim.2016.63019 


\section{Introduction}

$P$. aeruginosa is a gram-negative, aerobic, rod shaped bacterium with unipolar Motility, which is the main cause of prevalent hospital infections [1] [2]. It is recognized as a serious opportunistic pathogen that causes infections in hospitalized patients [3] [4]. The symptoms of these infections are generalized inflammation and sepsis. Particularly in burns patients where the skin host defenses are destroyed [5] [6], orthopedic related infection, respiratory disease [7], corneal infections caused by contact lens user [8], corneal ulceration [8], patient with impaired immune defense [9] [10] including HIV infection [11] [12] and, also is the most frequent colonizer in medical devices [13]. P. aeruginosa causes clinical problem as a result of its high resistance to a large rang of antibiotics [14] and may demonstrate additional resistance after unsuccessful treatment. Indeed treatment of $P$. aeruginosa infections in most cases should be conflicted, due to the resistance of these bacterial species and further acquired resistance to extensive range of antibiotics [3]. P. aeruginosa frequently displays resistance to multiple antimicrobial agents [15] [16]. Gentamycine is the only antibacterial agent through which pseudomonas strains are remains sensitive against it. There are different ranges of cross resistance between Carbencilin, Polymaxin, Colistin, Streptomycin, Quinolones, Cephalosporin against it reported [14] [17]. Therefore, antimicrobial resistance is a growing problem worldwide, especially in hospitals. Serious infections due to $P$. aeruginosa strains that exhibit resistance patterns to most common antibacterial agents are increasingly serious problems [16]. In this regard, the Pseudomonas areuginosa resistance pattern against antibiotics has not been studied in Urmia, Iran. We aimed to determine sensitivity and resistance patterns of $P$. aeruginosa against various antibiotics among clinical samples of educational Hospitals of Iran, Urmia University of medical science in western of Iran.

\section{Material and Methods}

\subsection{Study Setting}

A cross-sectional study during March 2009 to September 2011was performed. This study was conducted in the Internal, surgery, burns and transplantation ward of educational hospitals of Urmia University of medical science, Iran. Indeed we examined 233 clinical samples were collected from different clinical sampling from wound swab, urine samples, blood, trachea samples, eye swabs, ear swabs and pus from their patients then examined. All procedures of this investigation performed with the approval of the scientific and ethical board of this college, and patient consent form signed with each subjects for permission to use of their samples as case of study.

Isolation and identification of Pseudomonas aeroginousa

For these sample processing, in sterill condition, Nutrient agar, Mac Conkey agar and blood agar used for each samples. All plates incubated at $37^{\circ} \mathrm{C}$ for $24 \mathrm{~h}$. After incubation periodto obtain pure cultures according to Chessborough (Cheesborough 2002 based protocol), single pure colonies were subcultured. Then pure samples used To isolation and Different identification tests of Pseudomonas aeruginosa strains using macroscopic and microscopic biochemical methods Based on Bergey's manual of determinative bacteriology that describes Pseudomonas aeruginosa Oxidative and non-fermentative strain, with positive oxidase reaction, citrate utilization test, production of green pigment (Pyocyanin) on Mueller-Hinton agar medium.

\subsection{Antibiotic Susceptibility Test}

Antibiotics MIC with Agar dilution methods was determined. In this way isolates based on disk diffusion method (using Kirby-baur method) with common and valid Antibiotics on Mueller-Hinton agar (Mueller-Hinton agar Formula/Liter: Beef Extract: 2 g, Acid Hydrolysate of Casein: 17.5 g, Starch: 1.5 g, Agar: 17 g, Final pH $7.3 \pm 0.1$ at $25^{\circ} \mathrm{C}$ ) medium, antibiotic susceptibility pattern according to Clinical Laboratory Standard Institute (CLSI) principals (NCCLS 2011) was determined [18]. Paper disk were smeary with antibiotics: Amikacin (30 ug), Gentomycin (10 $\mu \mathrm{g})$, Imipenem (10 $\mu \mathrm{g})$, Sparfloxacin $(5 \mu \mathrm{g})$, Ceftazidime (30 $\mu \mathrm{g})$, Piperacilin $(100 \mu \mathrm{g})$, Nalidik acid (30 ug), Ceftizoxime (30 $\mu \mathrm{g})$, Ceftriaxone (30 $\mu \mathrm{g})$, Carpencilin $(100 \mu \mathrm{g})$ and Trimtoprine $(5 \mu \mathrm{g})$ Solfametoxasole $(23.75 \mu \mathrm{g})$ and incubated at $37^{\circ} \mathrm{C}$ for 18 hours. We determined the diameter of zone of inhibition as sensitive and semi-sensitive and resistance patterns of $P$. aeruginosa against different antibioticsbased on CLSI guidelines (NCCLS 2011).

Statistical analysis

Statistical analysis performed with SPSS (ver.18) software, data were reported as mean \pm SD for continuous 
variables.

\section{Results}

Our study takes almost two years during March 2009 to September 2011.233 samples were isolated from wound swab, urine, trachea, eye swabs, ear swabs and pus of patients.

From Imam Khomeini Hospital, Urmia. Iran, Isolation rates of Pseudomonas aeruginosa according to departments presented in Table 1.

The mean age of the patients was $35.4 \pm 14.5$ years old and most of the patients were more than 34 years old. $81 \%$ were female and the remaining (18.2\%) were male. $20.9 \%$ patients were illiterate, $20 \%$ had studied until the elementary level, $11 \%$ attained guidance school, $12 \%$ attained high school, 35\% had diplomas, and 1.1\% patients had an academic degree. Data shown sixty samples were positive to Pseudomonas aeruginosa. Also 25.75\% of samples were resistant to all antibiotic, which the majority of them found in Transplantation ward. The resistance pattern against Cefotaxime (94.34\%), Ceftazidim (95.68\%), Nalidixic acid (95.32\%), Gentamycine (79.23\%), Carpencilin (81.54\%) and Piperacilin (94.37\%) was also observed in this study. The maximum antibiotic resistance 99.5\% was observed against Trimtoprine Solfametoxole. The most effective antibiotics (sensitive pattern) for P. aeruginosa were Ciprofloxacin (55.33\%), Amikacin (61\%) and Imipenem (33\%). Resistance pattern rate (\%) of Pseudomonas aeruginosa against antibiotics presented in Figure 1.

\section{Discussion}

P. aeruginosa emerged as an important pathogen and was reasonable for nosocomial infections. So, that is one of the important causes of morbidity and mortality among Hospital patients [3] [4] [19]. The current study showed that $P$. aeruginosa was highly prevalent in clinical samples of urine and pus, was also observed in Arshi, et al. and Murase et al. study [20] [21]. This study shows that $P$. aeruginosa resistant rate against Gentamycin was $79.23 \%$, while reports of the susceptibility of $P$. aeruginosa against Gentamycin in different studies have ranged from 49.8\%, in Greek to as high as $96.6 \%$ in the United Kingdom [22] and in another study, in 1988, from Turkey, it was reported that $54 \%$ of gram negative Bacilius were resistant to Gentamycin [23]. This survey shown

Table1. Isolation rates of Pseudomonas aeruginosa according to departments.

\begin{tabular}{lllllcccc} 
Samples & Wound samples & Urine samples & Trachea samples & Ear swabs & Eye swabs & Pus & Blood & Total \\
\hline Frequency & 20 & 102 & 12 & 25 & 6 & 20 & 233 & 48 \\
Percent (\%) & $8.58 \%$ & $43.78 \%$ & $5.15 \%$ & $10.73 \%$ & $2.57 \%$ & $20.7 \%$ & $8.25 \%$ & $100 \%$ \\
\hline
\end{tabular}

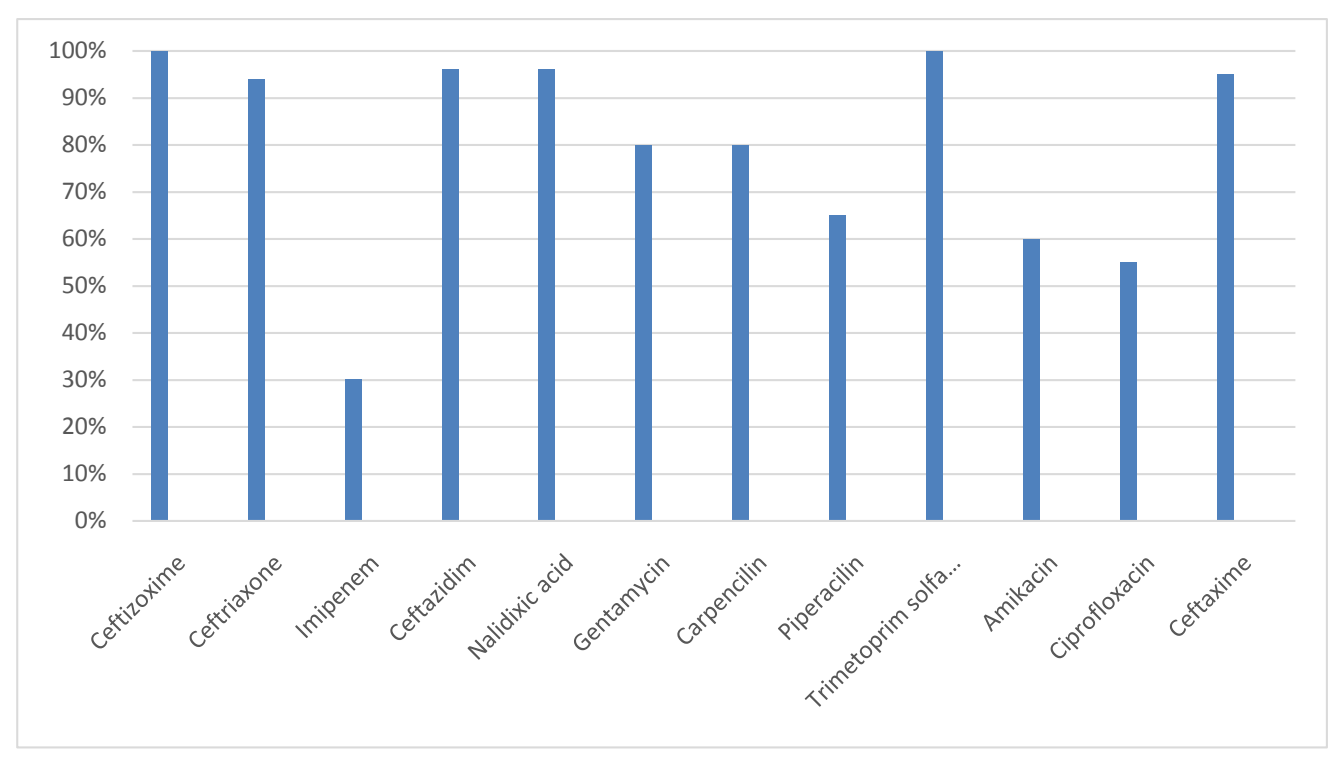

Figure 1. Resistance rate (\%) of Pseudomonas aeruginosa against Antibiotics. 
in our population study samples analysis revealed resistant patterns to Ceftazidime (95.67\%), while, according to different reports, resistance to Ceftazidime was $15 \%$ - $22 \%$ in the world, also Resistance to Ceftriaxime, Ceftriaxon and Nalidik acid are significant in our study (99.37, 94.37, 95.32 respectively). Previous studies suggested that selective pressure from the use of anti-microbial agents is a major determinant for the emergence of resistant strains [24]-[26] that could be considerable. Our results indicated that the rate of ciprofloxacin resistance pattern against $P$. aeruginosa was found to be relatively high percent in our population. Other studies show that ciprofloxacin resistance pattern was $23 \%$ in Spain [27], 31.9\% in Italy [28] and 26.8\% in Latin America [29]. Overall results show that the rate of $P$. aeruginosa antibiotic resistance was observed in highest rate in case of Trimetoprime Solfametaxasole, while, Ciprofloxacin, Imipenem and Amikacin were identified as the most effective antibiotics in this study against $P$. aeruginosa. In previous studies it was reported that resistance to Imipenem was 14\% in Spain [27], 19.3\% in Italy [28] and 68\% in Saudi Arabia [30] that approved with our results. Previous studies show that antibacterial effects of Amikacin against pseudomonal were greater than Gentamycin [23] [31] and only $0.9 \%$ resistant reported to Amikacin in Turkey [23]. So, Amikacin is effective antibiotic against $P$. aeruginosa in their study. In current study, resistance pattern to Piperacillin was also higher, while Piperacillin resistance rate was 10\% in Spain [27], 12\% in Italy [28], 14\% in Latin American population [29]. Indeed $P$. aeruginosa strains Infections, most be treated as soon as possible due to their severe resistance against antibiotics. We have to apply an accurate antibiotic treatment discipline, according to the findings based on antibiogram method in order to prevent its spread. Data showed that this bacterium has developed its resistance pattern against amikacin and Ciprofloxacin in comparison to previous years. So the use of effective infection control practices can go a long way to limiting the development and spread of antimicrobial resistance ensuring that these agents continue to find a place in treatment of $P$. aeruginosa infections and also, hospital infection monitoring committee needs to force the tasks of more severe monitoring on hospital pathogens and bacterial resistance pattern development studies as important objectives to finding effective antibiotics against $P$. aeruginosa.

\section{References}

[1] Ryan, K.J. and Ray, C.G. (Eds.) (2004) Sherris Medical Microbiology. 4th Edition, McGraw Hill, New York.

[2] Snell, K., Holderi, A., Lepplas, A. and Saelinger, C.B. (1978) Role of Exotoxin and Protease as Possible Virulence Factors in Experimental Infections with Pseudomonas aeruginosa. Journal of Clinical Microbiology, 9, 538-540.

[3] Echere, J.C. and Kohler, T. (1999) Patterns and Modes of $\beta$-Lactam Resistance in Pseudomonas aeruginosa. Clinical Microbiology and Infection, 5, S15-S18. http://dx.doi.org/10.1111/j.1469-0691.1999.tb00719.x

[4] Khan, J.A., Iqbal, Z., Rahman, S.U., Farzana, K. and Khan, A. (2008) Prevalence and Resistance Pattern of Pseudomonas aeruginosa against Various Antibiotics. Pakistan Journal of Pharmaceutical Sciences, 21, 311-315.

[5] Holder, I.A., Nathan, P. and Macmillan, B.G. (1973) Burn Wounds: Microbiology, Local Host Defences, and Current Therapy. Critical Reviews in Clinical Laboratory Science, 4, 61-100. http://dx.doi.org/10.3109/10408367309151684

[6] Erdem, B. (1999) Pseudomonas. In: Ustacelebi, S., Ed., Basic ClinMicrobiol., Gunes Publication, Ankara, $551-558$.

[7] Govan, J.R.W. (1992) Microbiology of Lung Infection in Cystic Fibrosis. British Medical Bulletin, 48, 912-930.

[8] Levinson, W. and Jawetz, E. (2000) Medical Microbiology and Immunology. 6th Edition, McGraw-Hill, New York, p. 123.

[9] Dark, J.K.G. (1988) Pathogenesis and Therapy of Pseudomonas aeruginosa. Eye, 2, 46-55. http://dx.doi.org/10.1038/eye.1988.133

[10] Smith, J., Payne, J. and Berne, T. (2000) The Surgeon's Guide to Antimicrobial Chemotherapy. CRC Press, Boca Raton, 38-74.

[11] O’Donnell, J.G., Sorbello, A.F., Condoluci, D.V. and Barnish, M.J. (1993) Sinusitis Due to Pseudomonas aeruginosa in Patients with Human Immunodeficiency Virus Infection. Clinical Infectious Diseases, 16, 404-406. http://dx.doi.org/10.1093/clind/16.3.404

[12] Flores, G., Stavola, J.J. and Noel, G.J. (1993) Bacteremia Due to Pseudomonas aeruginosa in Children with AIDS. Clinical Infectious Diseases, 16, 706-708. http://dx.doi.org/10.1093/clind/16.5.706

[13] Itah, A.Y. and Essien, J.P. (2005) Growth Profile and Hydrocarbonoclastic Potential of Microorganisms Isolated from Tarballs in the Bight of Bonny, Nigeria. World Journal of Microbiology and Biotechnology, 21, 1317-1322. http://dx.doi.org/10.1007/s11274-004-6694-z

[14] Adedeji, G.B., Fagade, O.E. and Oyelade, A.A. (2007) Prevalence of Pseudomonas aeruginosa in Clinical Samples and Its Sensitivity to Citrus Extract. African Journal of Biomedical Research, 10, 183-187. 
[15] Carmeli, Y., Troillet, N., Eliopoulos, G.M. and Samore, M.H. (1999) Emergence of Antibiotic-Resistant Pseudomonas aeruginosa: Comparison of Risks Associated with Different Anti Pseudomonal Agents. Antimicrobial Agents and Chemotherapy, 43, 1379-1382.

[16] Linden, P.K., Kusne, S., Coley, K., Fontes, P., Kramer, D.J. and Paterson, D. (2003) Use of Parenteral Colistin for the Treatment of Serious Infection Due to Antimicrobial-Resistant Pseudomonas aeruginosa. Clinical Infectious Diseases, 37, e154-e160. http://dx.doi.org/10.1086/379611

[17] Todar, K. (2002) Todar's Online Textbook of Bacteriology. pp. 1-8. http://textbookofbacteriology.net/nutgro.html

[18] Anab, F., Syed Baqir, N., Sheikh, A., Shaheen, P. and Sabahat, J. (2012) Antimicrobial Susceptibility Pattern of Clinical Isolates of Pseudomonas aeruginosa Isolated from Patients of Lower Respiratory Tract Infections. SpringerPlus, 1, 70.

[19] das Neves, M.T., de Lorenzo, M.E., Almeida, R.A. and Fotaleza, C.M. (2010) Antimicrobial Use and Incidence of Multidrug-Resistant Pseudomonas aeruginosa in a Teaching Hospital: An Ecological Approach. Revista da Sociedade Brasileira de Medicina Tropical, 43, 629-632. http://dx.doi.org/10.1590/S0037-86822010000600005

[20] Arshi, S., Manzoor, T., Syed, S. and Assadullah, S. (2007) In Vitro Sensitivity Pattern of Pseudomonas aeruginosa Strain Isolated from Patient Skims-Role of Antimicrobial in the Emergence of Multiple Resistance Strains. JK-Practitioner, 14, 31-34.

[21] Murase, M., Miyamoto, H., Handa, T., Sahaki, S. and Takenchi, N. (1995) Activities of Anti-Pseudomonal Agent against Clinical Isolates of Pseudomonas aeruginosa. The Japanese Journal of Antibiotics, 48, 1581-1589.

[22] Van Landuyt, H.W., Boelaert, J., Glibert, B., Gordts, B. and Verbruggen, A.M. (1986) Surveillance of Aminoglycoside Resistance. European Data. The American Journal of Medicine, 80, 76-81. http://dx.doi.org/10.1016/0002-9343(86)90483-3

[23] Alkalin, H.E., Torun, M. and Alacam, R. (1988) Aminoglycoside Resistance Patterns in Turkey. Scandinavian Journal of Infectious Diseases, 20, 199-203. http://dx.doi.org/10.3109/00365548809032438

[24] Jarvis, W.R. (1996) Preventing the Emergence of Multidrug Resistant Microorganisms through Antimicrobial Use Controls: The Complexity of the Problem. Infection Control and Hospital Epidemiology, 17, 490-495. http://dx.doi.org/10.2307/30141280

[25] Weber, D.J., Raasch, R. and Rutala, W.A. (1999) Nosocomial Infections in the ICU: The Growing Importance of Antibiotic Resistant Pathogens. Chest, 115, 34S-41S. http://dx.doi.org/10.1378/chest.115.suppl_1.34S

[26] Nathwani, D. (1998) Sequential Switch Therapy for Lower Respiratory Tract Infections: A European Perspective. Chest, 113, 211S-218S. http://dx.doi.org/10.1378/chest.113.3_Supplement.211S

[27] Bouza, E., Garcia-Gorrote, F., Cercenado, E., Marin, M. and Diaz, M.S. (1999) Pseudomonas aeruginosa: A Survey of Resistance in 136 Hospitals in Spain. The Spanish Pseudomonas aeruginosa Study Group. Antimicrobial Agents and Chemotherapy, 43, 981-982.

[28] Bonfiglio, G., Carciotto, V., Russo, G., Stefani, S., Schito, G.C., Debbia, E. and Nicoletti, G. (1998) Antibiotic Resistance in Pseudomonas aeruginosa: An Italian Survey. Journal of Antimicrobial Chemotherapy, 41, 307-310. http://dx.doi.org/10.1093/jac/41.2.307

[29] Jones, R.N. (2001) Resistance Patterns among Nosocomial Pathogens: Trends over the Past Few Years. Chest, 119, 397S-404S. http://dx.doi.org/10.1378/chest.119.2_suppl.397S

[30] Rotimi, V.O., Al-Sweih, N.A. and Feteih, J. (1998) The Prevalence and Antibiotic Susceptibility Pattern of Gram-Negative Bacterial Isolates in Two ICUs in Saudi Arabia and Kuwait. Diagnostic Microbiology and Infectious Disease, 30, 53-59. http://dx.doi.org/10.1016/S0732-8893(97)00180-6

[31] Maes, P. and Vanhoof, R. (1992) A 56-Months Prospective Surveillance Study on the Epidemiology of Aminoglycoside Resistance in a Belgian General Hospital. Scandinavian Journal of Infectious Diseases, 24, 495-501. http://dx.doi.org/10.3109/00365549209052636 